\title{
Efecto de un anticoccidial natural a base de saponinas de Yucca schidigera y Trigonella foenum-graecum sobre el control de coccidiosis en pollos de carne
}

\author{
Effect of a natural anticoccidial based on saponins of Yucca schidigera and \\ Trigonella foenum-graecum on the control of coccidiosis in broilers \\ Ingrid Rodríguez L. ${ }^{1}$, César Honorio J. ${ }^{1}$, Julia Ramírez S. ${ }^{2,3}$, Zara León G. ${ }^{2}$, \\ Willman Alarcón G. ${ }^{2}$
}

\section{Resumen}

\begin{abstract}
El objetivo del estudio fue evaluar el efecto de un anticoccidial natural a base de saponinas procedentes de Yucca schidigera y Trigonella foenum-graecum en el control de coccidiosis en pollos de carne. Se utilizaron 75 pollos machos de la línea Cobb 500 de un día de edad, distribuidos al azar en tres tratamientos: DBSA (dieta base sin adición de anticoccidial), DBAN (dieta base con adición de anticoccidial natural), DBQI (dieta base con adición de anticoccidial químico - ionóforo) con cinco repeticiones y cinco aves por unidad experimental. Los pollos fueron inoculados en día 14 con 15 veces la dosis recomendada de la vacuna viva (Coccivac-D), que contiene Eimeria acervulina, E. maxima, E. mivati, E. tenella, E. necatrix, E. brunetti, E. hagani y E. praecox para inducir la enfermedad. Se evaluaron las variables recuento de ooquistes por gramo de heces (ROpgh), lesiones intestinales y diversos parámetros productivos. Los valores de ROpgh fueron similares para DBAN y DBQI llegando a su pico a la séptima semana (328.8 y 455.8 Opgh, respectivamente), para luego disminuir. Las lesiones intestinales los días 10,20 y 28 pos-infección y los parámetros productivos al final del estudio fueron similares para ambos tratamientos, pero significativamente mejores que el control DBSA. Se concluye que las saponinas procedentes de $Y$. schidigera y $T$. foenum-graecum pueden remplazar eficientemente a los anticoccidiales químicos en la alimentación de las aves.
\end{abstract}

Palabras clave: coccidiosis; lesiones intestinales; saponinas; Yucca schidigera; Trigonella foenum-graecum

\footnotetext{
${ }^{1}$ Escuela Profesional de Veterinaria y Zootecnia, Universidad Privada Antenor Orrego, Trujillo, Perú

${ }^{2}$ Escuela Profesional de Zootecnia, Universidad Nacional de Trujillo, Trujillo, Perú

${ }^{3}$ E-mail:jramirez@unitru.edu.pe
}

Recibido: 21 de junio de 2018

Aceptado para publicación: 20 de diciembre de 2018 
The aim of this study was to evaluate the effect of a natural anticoccidial based on saponins from Yucca schidigera and Trigonella foenum-graecumen in the control of coccidiosis in broilers. It was used 75 1-day-old chicks from the Cobb 500 line, randomly distributed in three treatments: DBSA (base diet without addition of anticoccidial), DBAN (base diet with addition of natural anticoccidial), DBQI (base diet with addition of chemical anticoccidial - ionophore) with five repetitions and five chicks per experimental unit. The birds were inoculated on day 14 with 15 times the recommended dose of the live vaccine (Coccivac-D) which contains Eimeria acervulina, E. maxima, E. mivati, E. tenella, E. $n e c a t r i x, E$. brunetti, E. hagani and E. praecox to induce the disease. The variables oocyst count per gram of faeces (ROpgh), intestinal lesions and various productive parameters were evaluated. The values of ROpgh were similar for DBAN and DBQI peaking at the seventh week (328.8 and 455.8 Opgh, respectively), and then decreasing. Intestinal lesions on days 10, 20 and 28 post-infection and the productive parameters at the end of the study were similar for both treatments, but significantly better than the DBSA control. It is concluded that the saponins from Y. schidigera and T. foenumgraecum can efficiently replace chemical anticoccidials in bird feeds.

Key words: coccidiosis; intestinal lesions; saponins; Yucca schidigera; Trigonella foenum-graecum

\section{INTRODUCCIÓN}

La coccidiosis aviar es una enfermedad parasitaria cosmopolita frecuente, causada por protozoos del Phylum Apicomplexa, familia Eimeriidae. Aunque afecta a diversas especies de aves, es en el pollo de carne y en la gallina ponedora o reproductora donde alcanza la mayor repercusión económica. La infección se produce mediante la ingestión de ooquistes esporulados, que dan lugar a un proceso de carácter clínico o subclínico, caracterizado por diarrea y descenso de las producciones (Del Cacho, 2013).

En el control de la coccidiosis se utilizan vacunas mediante la administración oral de varias especies atenuadas de Eimerias (Alcaino et al. 2002), así como productos anticoccidiales. Dentro de estos últimos existen los químicos, que tienen mayor potencia de acción, y los ionóforos, que poseen una acción antibacteriana adicional que les permite actuar como promotor de crecimiento (Arnaiz, 2012); sin embargo, su uso está cuestionado debido a la resistencia que genera el protozoo frente a estos productos (Espejo, 2014), lo que ha llevado a la restricción en diversos países del uso de estos productos por producir efectos adversos en el ser humano (Revolledo, 2013). En 2003, la Unión Europea promulgó el Reglamento (CE) N. ${ }^{\circ}$ $1831 / 2003$ sobre los aditivos en la alimentación animal, estableciendo que a partir del 1 de enero de 2006 los antibióticos, coccidiostatos e histomoniatos no pueden ser usados en alimentos para animales, presentándose la necesidad de buscar nuevas alternativas.

Una alternativa a los coccidiostatos químicos son los extractos herbáceos (Chandrakesan et al., 2009). Entre los componentes de estos extractos se encuentran las saponinas, glucósidos esteroidales o triterpénicos que son generados principalmente por plantas, aunque también por bacterias y animales marinos menores (Nasri, 2011). Las saponinas tienen propiedades antibacterianas (especialmente bacterias Gram positivas como Bacillus cereus, B. subtilis, Staphylococcus aureus y 
Enterococcus faecalis), antiprotozoaria (especialmente sobre protozoos ruminales) y antifúngica (Saccharomyces cerevisiae, Candidaialbicans sp, Candidaiglabrata $\mathrm{sp}$, Candidaikrusei sp, Cryptococcus neoformans y Aspergillus fumigatus) (Espejo, 2014).

Las fuentes comerciales de saponinas son plantas del desierto como Yucca schidigera de México, Quillaja saponaria de Chile y Trigonella foenum-graecum (Cheeke, 2001). También se obtiene de la quinua (Chenopodium quinua) (Ahumada et al., 2016). Las saponinas son compuestos altamente lipofílicos y liposolubles, se desplazan a la membrana del parásito y se unen a un fosfolípido que es esencial para que el parásito ingrese a la célula intestinal del hospedador, formando un complejo y precipitándolo (Rambozzi et al., 2011; Espejo, 2014; Djezzar et al., 2014). Por esta razón, las saponinas son menos activas contra $E$. acervulina, dado que esta coccidia ingresa con más rapidez en la mucosa intestinal, quedando fuera de la acción del compuesto natural, que no se absorbe desde el intestino y solo actúa en la luz intestinal (Sims et al., 2001), por lo que las sapogeninas esteroidales son más efectivas contra $E$. tenella (Condemarín, 2002; Carranza et al., 2011).

Se ha demostrado que las saponinas de los extractos de Yucca schidigera y Trigonella foenum-graecum son efectivas en destruir las coccidias (Carranza et al., 2011), sumado a que son compuestos orgánicos prácticamente atóxicos, que no requieren de un periodo de restricción, no tienen efecto depresor del consumo de alimento y pueden usarse en múltiples combinaciones para programas duales, de allí que se evalúe su uso para el tratamiento de la coccidiosis en aves (Espejo, 2014). Por lo antes expuesto, se planteó el presente trabajo de investigación teniendo como objetivo evaluar los efectos de un anticoccidial natural a base de saponinas procedentes de Yucca schidigera y Trigonella foenum-graecum en el control de coccidias en pollos de carne.

\section{Materiales y Métodos}

La fase de campo se llevó a cabo en la granja «Olivares», ubicada en Trujillo, Perú. Se utilizaron 75 pollos machos de la línea Cobb 500 , de un día de edad (peso $50 \pm 3 \mathrm{~g}$ ) alojados en 15 corrales (repeticiones), adecuados con cama de viruta hasta el día 42. Todos los pollos se mantuvieron bajo las mismas condiciones de espacio y manejados de manera similar en cuanto a temperatura, ventilación, comederos, bebederos, alimentación y plan sanitario.

El peso se tomó los días 10, 22 y 42 por las mañanas, antes de que consuman alimento. El programa de vacunación incluyó enfermedades como Gumboro, Newcastle y bronquitis infecciosa. El alimento fue ofrecido de acuerdo con las etapas de inicio (22\% proteína cruda [PC] y $3015 \mathrm{kcal} / \mathrm{k} \mathrm{EM}$ ); crecimiento $(20 \% \mathrm{PC}, 3108 \mathrm{kcal} / \mathrm{k} \mathrm{EM})$ y acabado (19\% PC y $3180 \mathrm{kcal} / \mathrm{k}$ EM). La ración estuvo elaborada en base a maíz amarillo (Zea mays), torta de soya $45 \%$, soya integral y aceite de soya (Glicine max), aminoácidos y premezclas vitamínico mineral.

Los tratamientos fueron: DBSA: dieta base sin anticoccidial para las tres etapas; DBAQI: dieta base + anticoccidial químico (Clopidol 25\%, principio activo de Propidol25, Agrovet Market, Perú) para las etapas de inicio y crecimiento y anticoccidial ionóforo (Salinomicina 12\%, Chlorvet ${ }^{\circledR}$ Plus T. Montana, Perú) en la etapa de engorde; DBAN: dieta base + anticoccidial natural constituido por saponinas de los extractos de Yucca schidigera y Trigonella foenumgraecum, de nombre comercial Norponin XO (Nor-Feed, Francia) en las tres etapas. Las aves fueron inoculadas vía oral con la vacuna viva Coccivac-D (MSD) en el día 14, utilizando 15 dosis por ave con la finalidad de producir signos y lesiones por coccidiosis. $\mathrm{La}$ vacuna contiene $E$. acervulina ( $\geq 600$ oocistos), E. maxima ( $\geq 200$ oocistos), $E$. mivati ( $\geq 400$ oocistos), E. tenella ( $\geq 200$ oocistos), E. necatrix ( $\geq 400$ oocistos), E. 
brunetti ( $\geq 200$ oocistos), E. hagani $(\geq 400$ oocistos), E. praecox ( $\geq 400$ oocistos), según Espejo (2014).

Se hizo el recuento de ooquistes de Eimeria por gramo de heces (ROpgh) mediante análisis coproparasitológico. Para esto, se recolectaron heces del piso los días 7, 9, 11 y 13 pos-infección y se analizaron mediante la técnica de flotación (Gómez, 2010; Sixtos, 2010). Esta frecuencia fue debido a que, dependiendo de la especie, el ciclo de vida es de 4 a 7 días y la diseminación se efectúa por medio de heces, cama, polvo, escarabajos, moscas y otros fómites (Paredes y Quinteros, 2010); además, durante la fase endógena del parásito la producción de ooquistes se mantiene por varios días alcanzando un máximo durante los 7-8 días pos-infección (Conway y McKenzie, 2007). Así mismo, se sacrificaron dos aves por tratamiento los días 10,20 y 28 días pos-infección para evaluar las lesiones macroscópicas intestinales (Johnson y Reid, 1970), considerando la localización y la intensidad de las lesiones ( +0 : normal; +1 : infección ligera; +2 : infección moderada; +3 : infección grave; +4 : infección muy grave, con mortalidad). Los parámetros productivos evaluados fueron el incremento de peso (IP), el consumo de alimento (CA) y el índice de conversión alimenticia (ICA), obtenido con la fórmula ICA = alimento consumido / incremento de peso. Para el analisis estadístico se utilizó el análisis de varianza y la prueba de Tukey para hallar diferencias entre tratamientos. Se empleó el programa estadístico Infostat.

\section{Resultados}

\section{Recuento de Ooquistes}

El tratamiento Dieta base con adición de anticoccidial natural (DBAN) y el tratamiento Dieta base con adición de anticoccidial químico - ionóforo (DBAQI) presentaron recuentos bajos de ooquistes y sin diferencia significativa entre ellos. Por otro lado, los recuentos de ooquistes más altos se observaron en el tratamiento sin anticoccidial (DBSA), encontrándose diferencia significativa entre este tratamiento con respecto a los otros dos (DBAN y DBAQI) (Figura 1).

\section{Lesiones Intestinales}

Las calificaciones de lesiones intestinales se muestran en el cuadro 1, donde se aprecia que en todas las áreas del intestino en los tratamientos DBAN y DBAQI no se presentaron diferencias entre ellos y además obtuvieron las más bajas calificaciones. Por otro lado, el tratamiento DBSA obtuvo las calificaciones más altas.

\section{Parámetros Productivos}

Los promedios de los parámetros productivos se presentan en el Cuadro 2. Las ganancias de peso y consumo de alimento fueron similares entre los tratamientos DBAN y DBAQI y, como consecuencia, los índices de conversión alimenticia fueron también estadísticamente similares entre estos tratamientos. No obstante, el grupo de aves control (BDSA) fue significativamente diferente al tratamiento DBSA $(p<0.05)$.

\section{Discusión}

El tratamiento DBAN presentó los recuentos de ooquistes más bajos en comparación que el tratamiento químico (DBAQI) y el basal (DBSA), llegando a niveles bastante bajos en el día 13, al igual que el tratamiento DBAQI. Esto es posiblemente debido a que el ciclo completo de las coccidias en el intestino dura alrededor de siete días. Conway y McKenzie (2007) consideran que la producción de ooquistes se mantiene por varios días alcanzando un máximo durante los 7-8 días pos-infección, llegando a desaparecer a partir del día 12 , siempre y cuando no ocurra una reinfección. 


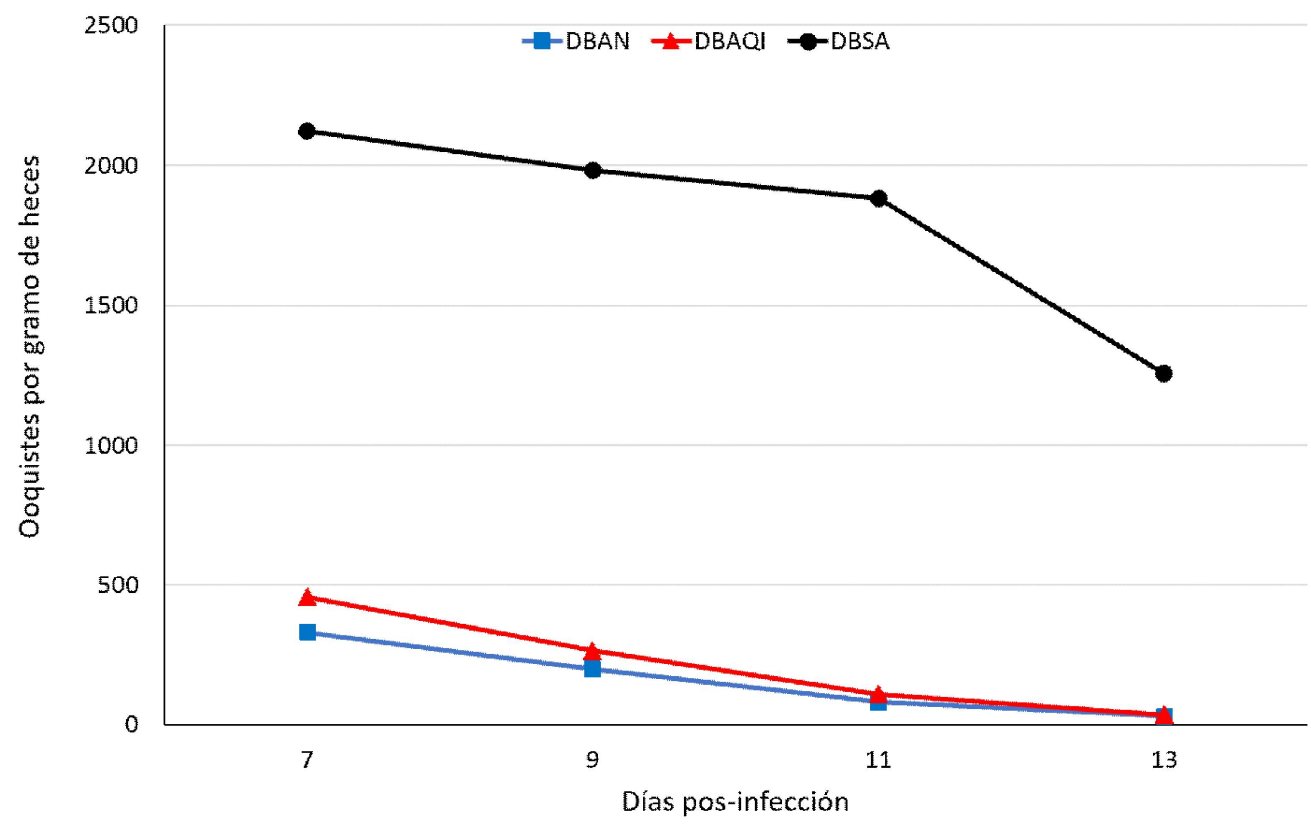

Figura 1. Ooquistes por gramos de heces (Opgh) de pollos de carne usando un anticoccidial natural a base de Yucca schidigera y Trigonella foenum-graecum, un anticoccidial químico - ionóforo y un grupo control sin anticoccidial DBAQI = Dieta base con adición de anticoccidial químico - ionóforo DBSA $=$ Dieta base sin anticoccidial DBAN $=$ Dieta base con adición de anticoccidial natural

La reducción de ooquistes se debería principalmente a la acción anticoccidial de la saponina. Rambozzi et al. (2011) indican que las saponinas por vía oral tienen efecto anticoccidial o anti-protozoario, debido a que se desplazan a la membrana del parásito y se unen a un fosfolípido que es esencial para que el parásito ingrese a la célula intestinal del hospedador, formando un complejo y precipitándolo. No obstante, al igual que el ionóforo, las saponinas no llegan a eliminar por completo a las coccidias. Carranza et al. (2011) encontraron recuentos de ooquistes similares a los del presente estudio, tanto en el tratamiento con saponinas como con el anticoccidial químico - ionóforo. Espejo (2014), por otro lado, en pollos alimentados con saponinas se observó el pico más alto de ooquistes al octavo día.
Salinas et al. (2001) y Long et al. (1975) consideran que la mayor concentración de ooquistes de Eimeria puede ser favorecida por las condiciones naturales de crianza, encontrando que el pico de recuentos de ooquistes se presentó mucho más tarde, entre las cuatro y las seis semanas posinfección; así mismo, Voeten (1987) refiere que la coccidiosis subclínica es más prominente a las 4-6 semanas de edad en el pollo si no se añaden anticoccidiales a la alimentación. En este tipo de coccidiosis los signos no son siempre evidentes y representa un riesgo permanente para la salud intestinal y el buen rendimiento productivo de las aves (Sumano y Gutiérrez, 2009).

Las calificaciones más altas de las lesiones intestinales corresponden a los conteos altos de ooquistes en el tratamiento DBSA, 
Cuadro 1. Promedio de calificación de lesiones intestinales ${ }^{1}$ en pollos de engorde usando un anticoccidial natural a base de Yucca schidigera y Trigonella foenum-graecum, un anticoccidial químico - ionóforo y un grupo control sin anticoccidial

\begin{tabular}{|c|c|c|c|c|c|}
\hline $\begin{array}{l}\text { Días pos- } \\
\text { infección }\end{array}$ & Órgano & DBAN & DBAQI & DBSA & E.E. \\
\hline \multirow[t]{4}{*}{ Día 10} & Intestino superior & $+1.6^{\mathrm{a}}$ & $+1.4^{\mathrm{a}}$ & $+2.6^{\mathrm{b}}$ & \\
\hline & Intestino medio & $+1.2^{\mathrm{a}}$ & $+1.4^{\mathrm{a}}$ & $+1.8^{\mathrm{a}}$ & 0.22 \\
\hline & Intestino inferior y recto & $+0.8^{\mathrm{a}}$ & $+1.0^{\mathrm{a}}$ & $+2.4^{\mathrm{b}}$ & 0.32 \\
\hline & Ciegos & $+1.0^{\mathrm{a}}$ & $+1.0^{\mathrm{a}}$ & $+1.6^{\mathrm{b}}$ & 0.14 \\
\hline \multicolumn{6}{|l|}{ Día 20} \\
\hline & Intestino superior & $+2.0^{\mathrm{a}}$ & $+2.2^{\mathrm{a}}$ & $+3.4^{\mathrm{b}}$ & 0.18 \\
\hline & Intestino medio & $+2.4^{\mathrm{a}}$ & $+2.6^{\mathrm{a}}$ & $+3.6^{\mathrm{b}}$ & 0.24 \\
\hline & Intestino inferior y recto & $+0.6^{\mathrm{a}}$ & $+0.8^{\mathrm{a}}$ & $+2.8^{\mathrm{b}}$ & 0.22 \\
\hline & Ciegos & $+1.4^{\mathrm{a}}$ & $+1.4^{\mathrm{a}}$ & $+2.4^{\mathrm{b}}$ & 0.24 \\
\hline \multirow[t]{4}{*}{ Día 28} & Intestino superior & $+2.0^{\mathrm{a}}$ & $+2.2^{\mathrm{a}}$ & $+4.0^{\mathrm{b}}$ & \\
\hline & Intestino medio & $+2.0^{\mathrm{a}}$ & $+2.0^{\mathrm{a}}$ & $+3.8^{\mathrm{b}}$ & 0.12 \\
\hline & Intestino inferior y recto & $0^{\mathrm{a}}$ & $0^{\mathrm{a}}$ & $+0.6^{\mathrm{b}}$ & 0.14 \\
\hline & Ciegos & $+0.2^{\mathrm{a}}$ & $+0.2^{\mathrm{a}}$ & $+2.4^{\mathrm{b}}$ & 0.22 \\
\hline
\end{tabular}

${ }^{a, b}$ Promedios con letras diferentes en la misma fila presentan diferencia significativa $(p<0.05)$

${ }^{1}$ Valores de clasificación según la descripción de Johnson y Reid (1970)

DBSA: dieta base sin anticoccidial para las tres etapas de crecimiento; DBAQI: dieta base + anticoccidial químico para las primeras dos etapas y anticoccidial ionóforo en la etapa de engorde; DBAN: dieta base + anticoccidial natural (saponinas de los extractos de Yucca schidigera y Trigonella foenum-graecum) en las tres etapas; E.E.: error estándar del promedio

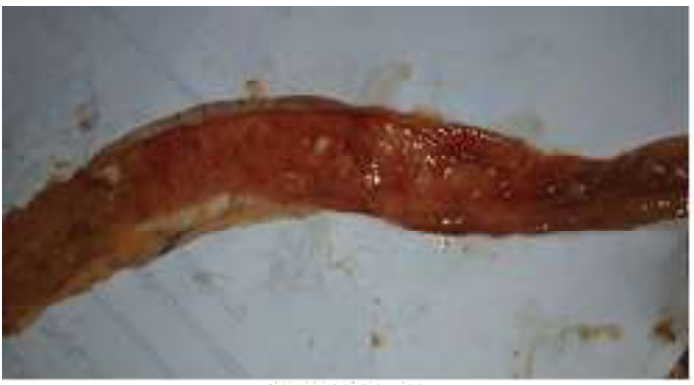

DBSA: +4

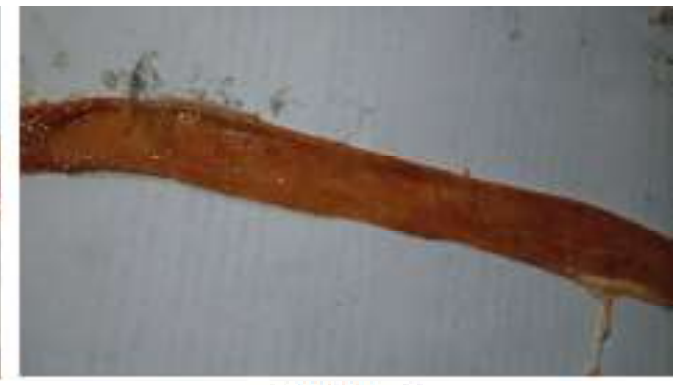

DBQI: +3

Figura 2. Lesiones observadas en epitelio del intestino medio de pollos de carne a los 20 días pos-infección con coccidias. La foto de la izquierda muestra el intestino de un ave del grupo control (sin anticoccidial). La foto de la derecha muestra el intestino de un ave tratada con un anticoccidial químico. Los grados de las lesiones corresponden a la clasificación de Johnson y Reid(1970) 


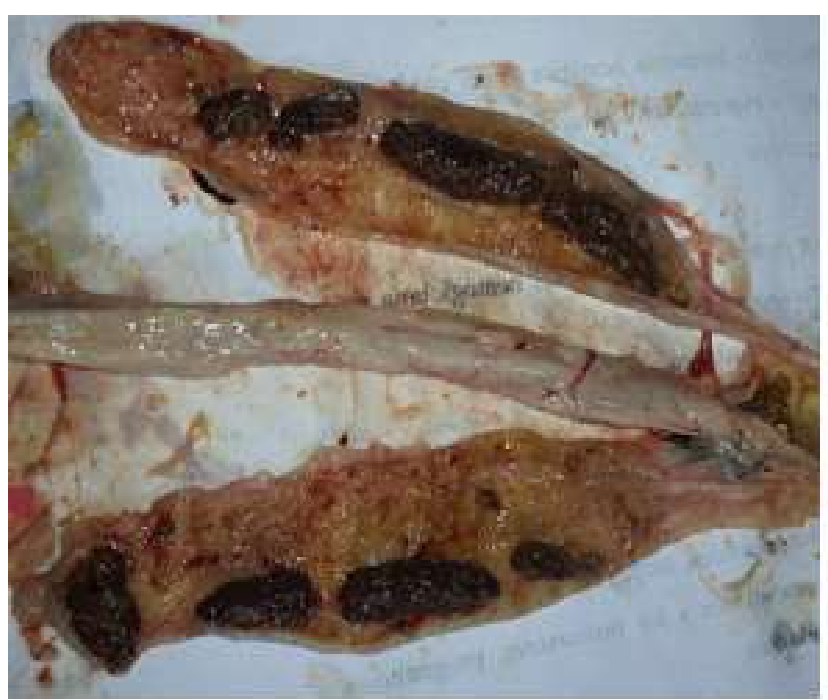

DBAN: +2

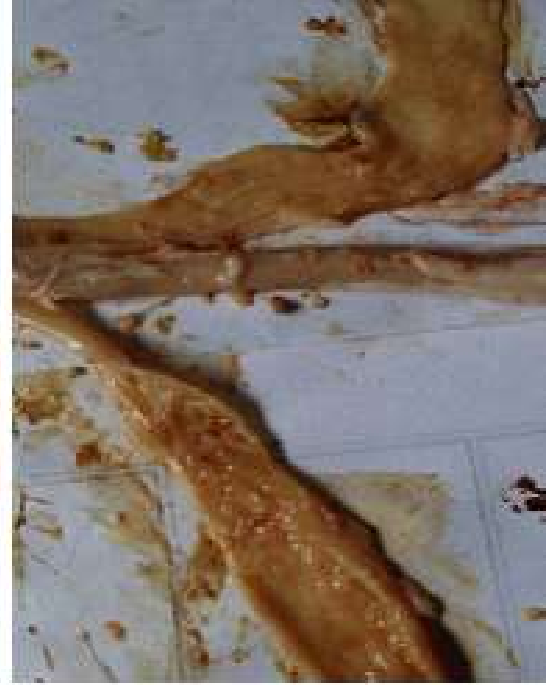

DBAN: +1

Figura 3. Lesiones observadas en el epitelio de los ciegos de pollos de carne a los 20 días posinfección con coccidias. La foto de la izquierda muestra el intestino de un ave tratada con un anticoccidial a base de saponinas. La foto de la izquierda muestra el intestino de un ave del grupo control (sin anticoccidial). Los grados de las lesiones corresponden a la clasificación de Johnson y Reid (1970)

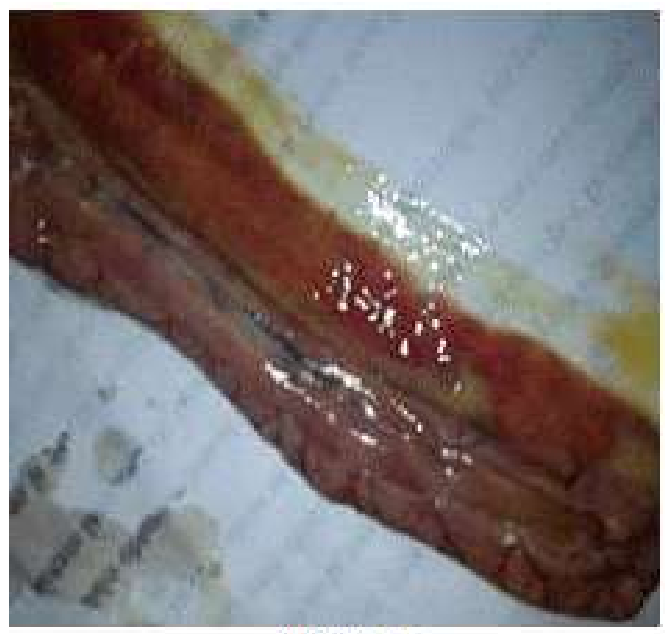

DBSA +4

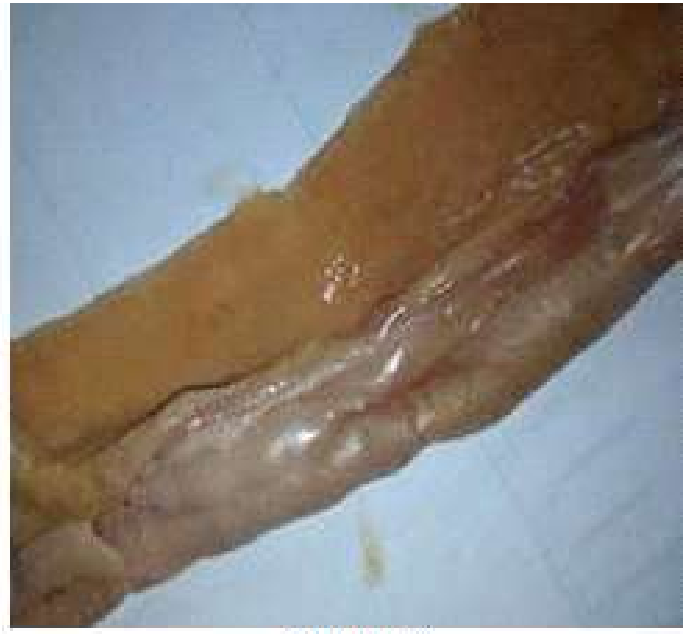

DBAN : +2

Figura 4. Lesiones observadas en el epitelio del intestino superior de pollos de carne a los 28 días pos-infección con coccidias. La foto de la izquierda muestra el intestino de un ave del grupo control (sin anticoccidial). La foto de la derecha muestra el intestino de un ave tratada con un anticoccidial a base de saponinas. Los grados de las lesiones corresponden a la clasificación de Johnson y Reid (1970) 
Cuadro 2. Parámetros productivos en pollos de engorde usando un anticoccidial natural a base de Yucca schidigera y Trigonella foenum- graecum, un anticoccidial químico - ionóforo y sin anticoccidial

\begin{tabular}{|c|c|c|c|c|}
\hline Fases / variables ${ }^{1}$ & DBAN & DBAQI & DBSA & E.E. \\
\hline \multicolumn{5}{|l|}{ Inicio } \\
\hline GP, $g$ & $232.3^{\mathrm{a}}$ & $223.5^{\mathrm{ab}}$ & $221.7^{\mathrm{b}}$ & 3.02 \\
\hline $\mathrm{CA}, \mathrm{g}$ & $283.4^{\mathrm{a}}$ & $275.1^{\mathrm{a}}$ & $273.4^{\mathrm{a}}$ & 3.43 \\
\hline ICA, $\mathrm{g} / \mathrm{g}$ & $1.22^{\mathrm{a}}$ & $1.23^{\mathrm{b}}$ & $1.23^{\mathrm{b}}$ & 0.00 \\
\hline \multicolumn{5}{|l|}{ Crecimiento } \\
\hline GP, $\mathrm{g}$ & $683.7^{\mathrm{a}}$ & $686.2^{\mathrm{a}}$ & $522.6^{\mathrm{b}}$ & 10.59 \\
\hline $\mathrm{CA}, \mathrm{g}$ & $930.3^{\mathrm{a}}$ & $944.7^{\mathrm{a}}$ & $831.1^{\mathrm{b}}$ & 7.26 \\
\hline ICA, $\mathrm{g} / \mathrm{g}$ & $1.36^{\mathrm{a}}$ & $1.39^{\mathrm{a}}$ & $1.61^{\mathrm{b}}$ & 0.02 \\
\hline \multicolumn{5}{|l|}{ Engorde } \\
\hline GP, $g$ & $2050.8^{\mathrm{a}}$ & $2034.1^{\mathrm{a}}$ & $1893.3^{b}$ & 41.07 \\
\hline $\mathrm{CA}, \mathrm{g}$ & $3808.8^{\mathrm{a}}$ & $3844.1^{\mathrm{a}}$ & $3605.0^{\mathrm{b}}$ & 39.14 \\
\hline ICA, $\mathrm{g} / \mathrm{g}$ & $1.88^{\mathrm{a}}$ & $1.89^{\mathrm{a}}$ & $1.90^{\mathrm{a}}$ & 0.03 \\
\hline \multicolumn{5}{|l|}{ Periodo total } \\
\hline $\mathrm{GP}, \mathrm{g}$ & $2970.3^{\mathrm{a}}$ & $2951.1^{\mathrm{a}}$ & $2644.8^{\mathrm{b}}$ & 51.64 \\
\hline $\mathrm{CA}, \mathrm{g}$ & $5023.4^{\mathrm{a}}$ & $5064.8^{\mathrm{a}}$ & $4711.0^{\mathrm{b}}$ & 47.92 \\
\hline ICA, $g / g$ & $1.70^{\mathrm{a}}$ & $1.72^{\mathrm{ab}}$ & $1.78^{\mathrm{b}}$ & 0.02 \\
\hline
\end{tabular}

que presentaron los cuatro tipos de lesiones desde los primeros días hasta el día 28 (Cuadro 1; Figuras 2, 3 y 4), Se observaron lesiones extremadamente severas en el intestino superior, ocasionadas por E. acervulina, resultados concordantes con Segovia-Gamboa et al. (2011). Estos autores afirman que $E$. acervulina se reproduce muy rápidamente, presentando cuatro generaciones de esquizontes y merozoítos en un 36-48 horas, pudiendo generar un número de ooquistes muy elevado.
Los tratamientos DBAN y DBAQI presentaron lesiones leves y moderadas en las secciones del intestino, sin diferencias significativas durante el tiempo de evaluación; sin embargo, no se observaron lesiones en el intestino inferior, ciego y recto el día 28 en ambos tratamientos, lo cual se debería a la acción de los anticoccidiales. No obstante, la parte media y superior del intestino presentaron valores que, a pesar de ser bajos $(+2)$, se mantuvieron durante el transcurso de las evaluaciones, concordante con el estudio de 
Adriano (2001), quien obtuvo calificaciones $\mathrm{de}+2$ en el intestino superior de pollos tratados con anticoccidial convencional. Así mismo, Condemarín (2002) encontró resultados similares, con mayor grado de lesiones en el duodeno de pollos tratados con sapogeninas, lo cual se relaciona con la menor acción de las saponinas sobre E. acervulina, debido a que esta ingresa con mayor rapidez a la mucosa y escapa a su acción. Por otro lado, Djezzar et al. (2014) obtuvieron resultados similares con puntuaciones inferiores $\mathrm{a}+2 \mathrm{en}$ pollos alimentados con saponinas.

Los resultados son concordantes con el estudio de Espejo (2014), quien observó que el extracto de saponinas del quillay (Quillaja saponaria) aplicadas a pollos de engorde mostraron un efecto de protección a la infección con coccidias. Hassan et al. (2008) demostraron igualmente que niveles altos de saponina natural (Gyamopsis tetragonoloba) fueron efectivos en reducir el número de ooquistes por gramo de deyecciones y capaces de prevenir la diarrea sanguinolenta producida por E. tenella en pollos infectados experimentalmente.

Los resultados del tratamiento DBAQI fueron concordantes a los hallazgos de Pérez (2015), quién desafió a un grupo de pollos con cepas de campo de tres especies de Eimeria y los suplementaron con un programa anticoccidial (Salinomicina/Nicarbazina), encontrando una significativa reducción de la mortalidad y signos clínicos por coccidiosis, y una significativa reducción del escore de lesiones intestinales macroscópicas de $E$. acervulina y E. máxima, mas no de $E$. tenella. A este respecto, en la presente investigación se pudo observar reducción de signos clínicos y ausencia de lesiones en los ciegos el día 28, lo que supone la ausencia o disminución de E. tenella. Smith y Strout (1979) en un estudio realizado con E. tenella también encontraron que los ionóforos provocaron inflamación y destrucción del esporozoito con ningún efecto aparente sobre la célula hospedadora. Sin embargo, en el presente estudio se obtuvo la mejor respuesta con el tratamiento con saponinas naturales (DBAN).

Respecto a los parámetros productivos (Cuadro 2), la respuesta productiva fue similar en los tratamientos DBAN y DBAQI, y con diferencias significativas $(\mathrm{p}<0.05)$ con el tratamiento DBSA para ganancia de peso, consumo de alimento e índice de conversión alimenticia; resultados similares a los reportados por Cabuk et al. (2004) y Cabuk y Condemarin (2011). Otero (2012), por otro lado, trabajando con cerdos tratados con saponinas procedente de Quillaja saponaria, obtuvo mejores ganancias de peso, velocidad de crecimiento y menor acumulación de grasa abdominal. Begum et al. (2015) encontraron, asimismo, que la adición de Yucca schidigera en la dieta de pollos de engorde mejoró la ganancia de peso y el índice de conversión alimenticia, indicando que estos resultados pueden deberse a la mejora de la absorción de nutrientes por el aumento de la permeabilidad intestinal a través de la despolarización de las membranas.

Los resultados comparados con los valores referenciales del Manual Cobb 500 (2015), indican que los tratamientos DBAN y DBAQI presentaron valores más cercanos a los referenciales. Igualmente el mejor beneficio costo se obtuvo en los tratamientos que recibieron los anticoccidiales, especialmente el que recibió las saponinas.

\section{Conclusión}

El anticoccidial natural a base de saponinas procedentes de Yucca schidigera y Trigonella foenum-graecum controló la infección producida por coccidios, disminuyendo el número de ooquistes en heces, controló las lesiones intestinales y mejoró los parámetros productivos, presentando similar efecto anticoccidial al ionóforo químico salinomicina. 


\section{Literatura Citada}

1. Ahumada A, Ortega A, Chito D, Benitez R. 2016. Saponinas de quinua (Chepodium quinoa Willd.): un producto con alto potencial biológico. Rev Colomb Cienc Quím Farmacéuticas 45: 438-469. doi: 10.15446/rcciquifa.$\mathrm{v} 45 \mathrm{n} 3.62043$

2. Alcaíno H, González JP, Fredes F, Gorman T. 2002. Coccidias aviares de gallineros industriales de Chile. Parasitol Latinoam 57: 34-39. doi: 10.4067/S071777122002000100009

3. Arnaiz V. 2012. Programa de promotores y anticoccidiales: visión de un nutricionista. En: XV Reunión de la Asociación de Médicos Veterinarios Especialistas en Avicultura del Ecuador (AMEVEA). Quito, Ecuador.

4. Begum M, Hossain M, Kim I. 2015. Effects of caprylic acid and Yucca schidigera extract on growth performance, relative organ weight, breast meat quality, haematological characteristics and caecal microbial shedding in mixed sex Ross 308 broiler chickens. Vet MedCzech 11: 635-643. doi: 10.17221/8532VETMED

5. Cabuk M, Alcicek A, Bozurt M, Akkan S. 2004. Effect of Yucca schidigera and natural Zeolite on broiler performance. Int J Poultry Sci 3: 651-654. doi: 10.3923/ijps.2004.651.654

6. Carranza V, Orozco J, Urribe JJ, Fuentes VO, Taylor A. 2011. Uso de sapogeninas esteroidales en la prevención de coccidiosis aviar. Nota técnica. Rev Cient-Fac Cien V 21: 115-117.

7. Chandrakesan P, Muralidharan VD, KuMar G, Ponnudurai TJ, Harikrishnan K, Rani V. 2009. Efficacy of a herbal complex against caeca coccidiosis in broiler chickens. Vet Arhiv 79: 199-203.

8. Cheeke P. 2001. Actual and potential applications of Yucca shidigera and Quillaja saponaria saponins in human and animal nutrition. In: Oleszek W, Marston A (eds). Saponins in food, feedstuffs and medicinal plants. Kluwer Academic Publishers. p 241-254.

9. Condemarín A. 2002. Rendimiento productivo de pollos de carne criados con el anticoccidial natural: sapogeninas esteroidales. Tesis de Médico Veterinario. Lima, Perú: Univ. Nacional Mayor de San Marcos. 43 p.

10. Conway DP, Mckenzie E. 2007. Introduction to coccidiosis. In: Poultry coccidiosis. $3^{\text {rd }}$ ed. Iowa, USA: Blackwell Publishing. p 7-10.

11. Del Cacho E. 2013. Coccidiosis: la enfermedad, consecuencias y tratamiento. En: 50 Congreso Científico de Avicultura. Zaragoza, España.

12. Djezzar R, Benamirouche K, BaazizeAmmi D, Mohamed-Said R, Guetarni D. 2014. Effect of a dietary supplementation combining a probiotic and a natural anticoccidial in broiler chickens. Afr J Agr Res 9: 3782-3788. doi: 10.5897/ AJAR2014.8466

13. Espejo R. 2014. Evaluación experimental da las saponinas del Quillay (Quillaja saponaria) como inhibidoras del desarrollo de coccidias intestinales en pollos de engorda. Tesis de Médico Veterinario. Santiago de Chile: Univ. de Chile. 71 p.

14. Gómez C. 2010. Diagnostico parasitario en los animales del centro de rescate de fauna silvestre Yana Cocha, ciudad de Puyo, provincia de Pastaza. Tesis de Médico Veterinario. Ecuador: Univ. Técnica de Cotopaxi. $50 \mathrm{p}$.

15. Hassan S, El-Gayar A, Cadwell D, Bailey C, Cartwright A. 2008. Guar meal ameliorates Eimeria tenella infection in broiler chicks. Vet Parasitol 157: 133-138. doi: 10.1016/j.vetpar.2008.07.005

16. Johnson J, Reid WN. 1970. Anticoccidial drugs: lesion scoring technics in battery and floor-pen experiments with chickens. Exp Parasitol 28: 30-36. doi: 10.1016/0014-4894(70)90063-9 
17. Long PL, Tompkins RV, Millard BJ. 1975. Coccidiosis in broilers; evaluation of infection by the examination of broiler house litter for oocystos. Avian Pathol 4: 297-289. doi: 10.1080/03079457509353877

18. Nasri S, Salem HB, Vasta V, Abidi S, Makkar HPS, Priolo A. 2011. Effect of increasing levels of Quillaja saponaria on digestion, growth and meat quality of Barbarine lamb. Anim Feed Sci Tech 164: 71-78. doi: 10.1016/ j.anifeedsci.2010.12.005

19. Otero $W$. 2012. Efecto de la saponina hibotek (Quillaja saponaria) en los alimentos de cerdos en las etapas de crecimiento y engorde. Tesis de Ingeniero Zootecnista. Riobamba, Ecuador: Escuela Superior Politécnica de Chimborazo. $55 \mathrm{p}$.

20. Paredes E, Quinteros P. 2010. Determinación de la prevalencia de coccidiosis en las granjas avícolas de la parroquia Imbaya del cantón Antonio Ante y estudio del desempeño de tres tipos de drogas anticoccidiales en el control de protozoarios del género Eimeria durante la producción de pollos parrilleros. Tesis Ingeniero Agropecuario. Ibarra, Ecuador: Pontificia Universidad Católica del Ecuador. 109 p.

21. Perez JA. 2015. Escore de lesiones intestinales macroscópicas de coccidias en pollos de engorde desafiados con cepas locales de Eimerias y suplementados con un programa anticoccidial (salinomicina/ nicarbazina). Tesis de Médico Veterinario. Lima, Perú: Univ. Nacional Mayor de San Marcos. 61 p.

22. Rambozzi L, Molinar R, Menzano A. 2011. In vivo anticoccidial activity of Yuca schidigera saponins in natural infected calves. J Anim Vet Adv 10: 391394. doi: 10.3923/javaa.2011.391.394

23. Reglamento (CE) $N^{\circ} 1831 / 2003 \mathrm{del}$ Parlamento Europeo y del Consejo. 2003. Diario Oficial de la Unión Euro- pea. [Internet]. Disponible en: https:// www.boe.es/doue/2003/268/L0002900043.pdf

24. Revolledo L. 2013. Algunas alternativas a los anticoccidianos en el tratamiento de la coccidiosis aviar. [Internet]. Disponible en: Actualidad Avipecuaria. http:/ /www.actualidadavipecuaria.com/ articulos/alternativas-anticoccidianos-tratamiento-de-la-coccidiosis-aviar

25. Salinas M, Icochea E, Casas E, Falcón N, Reyna P. 2001. Niveles de ooquistes de Eimeria en cama y su relación con las lesiones intestinales en pollos broiler. Rev lnv Vet Peru 12: 8-13. doi: 10.15381/rivep.v12i1.7418

26. Segovia-Gamboa N, TalamásRohana $P$, Ángel-Martínez A, Cázares-Raga, F, et al. 2011. Differentiation of Entamoeba histolytica: a possible role for enolase. Exp Parasitol 129: 65-71.

27. Sims MD, Mathis GF, Walter RD. 2001. Safety evaluation of the anticoccidial CocciGuard in broiler chickens. In: Concurrent Meetings of The Southern Poultry Science Society, 22 ${ }^{\text {nd }}$ Annual Meeting of the The Southern Conference on Avian Diseases, $42^{\text {nd }}$ Annual Meeting. USA.

28. Sixtos C. 2010. Procedimientos y técnicas para la realización de estudios coproparasitoscópicos. Virbac al Día 3: 6-7.

29. Smith CK, Strout RG. 1979. Eimeria tenella accumulation and retention of anticoccidial ionophores by extracellular sporozoites. Exp Parasitol 48: 325-330.

30. Sumano H, Gutierrez L. 2009. Antiparasitarios. En: Farmacología clínica en aves. $3^{\circ}$ ed. México: Ed Interamericana. p 365-454.

31. Voeten AC. 1987. Coccidiosis: a problem in broilers. In: Verstegen MW, Henken AM (eds). Energy metabolism in farm animals: effect of housing, stress and disease. Dordrecht, The Netherlands: Martinus Nijhofflishers. p410-422. 